\title{
Conducta autolítica y parasuicida. Características sociodemográficas en población infantojuvenil de ámbito rural
}

RESUMEN: Este estudio realiza la descripción de los factores sociodemográficos y de las características formales asociadas a las tentativas suicidas en población infanto-juvenil de ámbito rural. La muestra la componen 33 sujetos (6 varones y 27 mujeres) con edades comprendidas entre los 7 y 24 años, que son atendidos en un Equipo de Salud Mental durante el año 2001, por haber realizado una tentativa suicida. El objetivo de la investigación es la descripción de factores de riesgo asociados que estén relacionados con el intento de suicidio para la identificación de población de riesgo así como para la prevención de nuevas tentativas. Los resultados obtenidos coinciden con los señalados por la literatura especializada en los aspectos principales. Señalando a las mujeres entre 14 - 17 años con sintomatología afectiva y con conflictos interpersonales como la población de mayor riesgo de realizar una tentativa suicida, siendo el método más utilizado la sobreingesta de fármacos.

PALABRAS CLAVE: conducta autolitica y parasuicida, infantil, adolescente, jóvenes adultos, ámbito rural

\section{Introducción.-}

Los comportamientos autodestructivos siempre han generado sorpresa y estupor, tanto en los profanos, como en los estudiosos del comportamiento humano, recibiendo la atención de diferentes
ABSTRACT: This study described the social-demography factors and formal characteristics associated to the suicidal attempts in childhood and adolescent population of rural ambit. The sample is composed by 33 subjects (6 male and 27 female) in the range of 7 and 24 years, attend in Mental Health Equipment during 2001 with motive of suicidal attempt. The objective of this study is the description of risk factors associated and the related aspect with the suicidal attempt for the identification the risk population as preventive measurement of new tentatives. The obtain issues coincided with the show to specialised literature which identify to the high risk population suicidal tentative in female between 14-17 years because of overingestion of medicament a low deadlines with affectives symptoms and interpersonal conflicts as next factor. We discussed the obtain result and established the necessity of more specific study for most outstanding variables of study.

KEYWORDS: suicidal and parasuicidal behaviour, childhood, adolescent, adult young, rural ambit

disciplinas (sociología, psicología,, medicina, religión, literatura, etc...), lo que nos da idea de su complejidad y constante presencia en el devenir de la humanidad. Dicho comportamiento, adquiere dimensiones mayúsculas cuando ocurre en Rev. Asoc. Esp. Neuropsiq., 2002, vol XXI, n. ${ }^{\circ} 84$, pp. 49-64 
niños y jóvenes, convirtiéndose en una experiencia conmovedora, difícil de aceptar y comprender.

En los últimos años, ha habido un aumento de los fenómenos suicidas en jóvenes $(1,2)$, estando presente en el $40 \%$ de los ingresos paido-psiquiátricos urgentes (3) y pasando a ser la segunda causa de muerte en la adolescencia.

Son numerosos los estudios realizados sobre la población infantil y adolescente $(4,5,6,7)$ y aunque se encuentran algunas conclusiones en las que los diferentes autores se muestran de acuerdo, existen todavía diversos aspectos que permanecen sin consenso, posiblemente por las dificultades metodológicas asociadas a la selección de la muestra y recogida de los datos.

En estos estudios, encontramos dos aproximaciones teóricas fundamentales del acto suicida. Para algunos autores sería un "acto continuo y progresivo", que engloba la ideación, los gestos, la tentativa suicida y el suicidio consumado $(8,9)$, y para otros, las ideas y los comportamientos suicidas son actos diferenciados, discontinuos y explicados por variables distintas (10).

Siguiendo a Mardomingo (11) "el suicidio consumado engloba todos aquellos actos lesivos auto-inflingidos con resultado de muerte, el intento de suicidio se define como el acto por medio del cual un individuo de forma deliberada se inflinge daño a si mismo sin resultado de muerte, independientemente de si existe deseo o intención de morir, cuando no existe tal deseo se le denomina gesto suicida". La ideación suicida se refiere a cogniciones que pueden variar desde pensamientos fugaces de que la vida no vale la pena vivirla, hasta imágenes autodestructivas persistentes y recurrentes (12). Los pensamientos suicidas preceden a los actos suicidas y muchos suicidios consumados están precedidos de intentos previos (1). Estos autores señalan como factor de riesgo cualquier conducta del espectro suicida. Otros sin embargo, diferencian la población que realiza un intento auto-lítico impulsivo, no premeditado y con baja probabilidad de consumarlo de los que realizan uno premeditado e intencionado (13). En el primero no habría trastorno psiquiátrico asociado, ni graves problemas en el medio familiar y sería posible identificar un factor desencadenante, mientras que en el segundo habría trastornos psiquiátricos, un medio familiar conflictivo y no sería fácil identificar un factor desencadenante.

Los datos epidemiológicos sobre las tasas de suicidio y las tentativas suicidas son poco fiables en los grupos menores de edad, posiblemente por la dificultad de definir este acto en la infancia. Entre los 5-14 años para la población USA en 1991, la incidencia fue de 0,7 por 100.000 habitantes. Los datos de la OMS oscilan entre 0 y 1,1 por 100.000 habitantes. En nuestro país, Gastaminza (14) refiere que el $18 \%$ de las tentativas de suicidio ocurre en menores de 12 años. En el rango entre 15-24 años para la población USA en 1991, se registró una tasa de 13,1 por 100.000 habitantes, siendo la segunda causa de muerte en este tramo de edad. Los datos de la OMS, para el mismo grupo, oscilan entre 1,1 y el 17,2 por 100.000 habitantes. Siendo mucho mayor la tasa para los varones (61 por 100.000 habitantes) frente a la tasa para las mujeres (5 por 100.000 habitantes). Así como 
el suicidio consumado es más frecuente en los hombres que en las mujeres, con una proporción de 10 a 3, las tentativas suicidas son más frecuentes en las chicas con una proporción de 9 a 1 .

Los datos indican un incremento de las conductas suicidas en las últimas décadas. Los datos del Instituto Nacional de Estadística (INE) para el último quinquenio (1996-2001) (15) en la población española, presenta una tasa de suicidio de 6,35 por 100.000 habitantes, oscilando para las diferentes provincias entre el 2,39 de Las Palmas y el 15,24 por 100.000 habitantes de Lugo. Para la provincia de Córdoba, de donde se selecciona la muestra de este estudio, es de 9,92 por 100.000 habitantes.

Los factores de riesgo asociados a la conducta suicida en la infancia y adolescencia han sido numerosos, encontrándose en ocasiones datos contradictorios. Pasamos a describir brevemente los modelos teóricos y resumir los datos más significativos encontrados en los diferentes estudios revisados.

El suicidio es el resultado de la confluencia de multitud de situaciones y factores que se combinan entre sí para generar un abanico de conductas suicidas que irían desde la simple ideación hasta el suicido consumado (16). Las variables que influyen en la conducta suicida son numerosas e incluyen factores genéticos, biológicos, sociodemográficos, psiquiátricos y psicosociales. Autores como Blumethal y Kupfer (17), proponen un modelo explicativo de la conducta suicida basado en la interacción de los factores de riesgo, que agrupan en cinco dominios (biológicos, rasgos de personalidad, trastornos psiquiátricos, familiares y genéticos y psicosociales). Siendo mayor la probabilidad de conducta suicida según se incrementa la presencia de factores de riesgo en los diferentes dominios. A su vez, estos factores los diferencian en distales y proximales. Los distales se relacionan con la vulnerabilidad al acto suicida, mientras los proximales estarían relacionados directamente con este. De la combinación de ambos tipos de factores se explicaría el acto suicida.

Respecto a los factores estudiados que tienen una mayor presencia en la población de niños y jóvenes destacan dentro de los sociodemográficos: la edad, siendo la tentativa suicida más frecuente en los jóvenes, en el tramo de edad entre los 15-24 años (18); con respecto al sexo los varones cometen un mayor número de suicidios consumados (19) y las mujeres mayor numero de tentativas (20). Respecto a los factores climáticos no se han obtenido datos concluyentes, siendo contradictorios entre los diferentes estudios, aunque si se puede resaltar una mayor incidencia en los meses de primavera-verano (21). Con respecto al día de la semana, tampoco existen datos concluyentes, señalando algunos estudios como dato significativo una menor incidencia de ocurrencia en los fines de semana (22). Por otro lado, el estado civil es un dato concluyente en la población adulta, siendo el matrimonio un factor de protección, mientras en la población adolescente el estar casado actúa como factor de riesgo (23). Respecto a la influencia de los acontecimientos vitales sobre la conducta suicida si se obtienen datos concluyentes, confirmándose la elevada incidencia de acontecimientos vitales en los meses previos a la conducta 
suicida $(23,24)$, esta sucesión de acontecimientos vitales en los meses previos a la tentativa ha sido denominada por Arranz (16) como "carrera suicida". En cuanto a los tipos de estresores identificados en los suicidas jóvenes encontramos la ocurrencia de alguna "perdida", los conflictos interpersonales, la separación, los conflictos económicos y los legales (25). La influencia de haber estado expuesto a un modelo suicida teniendo conocimiento del mismo, de forma directa o a través de los medios de comunicación, incrementa el riesgo en las personas predispuestas al suicidio y preferentemente en la población adolescente (26). Otros de los aspectos mas estudiados es la conflictividad en el medio familiar (3), encontrándose como desencadenante las disputas con los padres. Por otro lado, uno de los aspectos más señalados en la literatura científica es la ausencia del padre en el medio familiar (3). La existencia de patología mental asociada (27), ha sido una de las variables que ha obtenido más resultados significativos en los diferentes estudios, encontrándose altas correlaciones con trastornos afectivos, trastornos de conducta y trastornos de adaptación. En esta línea, los datos de tentativas de suicidio son muy superior en la población psiquiátrica comparados con la población normal (28). Respecto al método más utilizado en la población de jóvenes es, sin duda, la sobreingesta medicamentosa la que ocurre en una mayoría de los casos $(14,29)$.

El conocimiento de los factores asociados al comportamiento suicida y la identificación de sujetos en situación de riesgo son las medidas preventivas más utilizadas (30). Los estudios realizados ponen de manifiesto un alto porcentaje de repetición de las conductas parasuicidas en adolescentes, siendo uno de los predictores más potentes de ocurrencia de nuevas tentativas y de suicidio consumado (31). El pronóstico de los sujetos es peor si no se encuentran en tratamiento (29). Debido a la escasez de investigaciones en la población infanto-juvenil de ámbito rural y más concretamente de la localidad donde realizamos este estudio, el objetivo fundamental de este, es la obtención de datos para la identificación de factores de riesgo en población infanto-juvenil para tener un mejor conocimiento de la población que realiza una tentativa de suicidio, identificándolos como sujetos de alto riesgo y promover medidas preventivas para la disminución de la repetición de las mismas.

\section{Método}

Sujetos

La población objeto de estudio la forman 33 sujetos atendidos en el Equipo de Salud Mental de Montilla (dispositivo extrahospitalario dependiente del Hospital Reina Sofía de Córdoba), representando el total de la población infanto-juvenil (hasta $\operatorname{los} 24$ años) remitida por los servicios de urgencias y atención primaría por intento autolítico y conducta parasuicida, durante el año 2001, independientemente del riesgo letal del intento. El total de la población atendida en el mismo equipo para el mismo rango de edad y por otros motivos clínicos es de 348 pacientes, por lo que la población que realiza una tentativa suicida representa el $10 \%$ de la población atendida, y el $60 \%$ de las consultas urgentes para el mismo rango de edad. El grupo está formado por 6 varones y 27 mujeres con un rango de edad comprendido entre los 7 y los 24 años. 


\section{Material}

El instrumento básico para recoger la información que, posteriormente, se utiliza para el análisis estadístico es la entrevista clínica semiestructurada, tradicionalmente utilizada en los Equipos de Salud Mental del Servicio Andaluz de Salud (S.A.S.), de la cual se extraen datos clínicos relacionados con la conducta suicida y los datos epidemiológicos básicos centrados en variables sociodemográficas y personales se obtienen del programa SISMA V. 5.0. de gestión de Historia Clínicas, utilizado en los Equipos de Salud Mental del Servicio Andaluz de Salud (S.A.S.).

Para el análisis estadístico descriptivo utilizamos el paquete estadístico SPSS, versión 10.1, Módulo BASE.

\section{Procedimiento}

Tras un análisis preliminar de las historias clínicas correspondientes, seleccionamos variables epidemiológicas básicas y otras variables específicas, relacionadas en la literatura con el suicidio, para su posterior análisis estadístico.

Seleccionamos el sexo del sujeto, con dos opciones: hombre y mujer; edad en la que se produce el intento (o en el caso de haber varios, el último intento); estudios, con cinco niveles: primaria, secundaria, bachiller, F.P., universidad; número de hermanos; estado civil de los padres con dos niveles: casados y otras situaciones; antecedentes psiquiátricos familiares con nueve niveles: trastorno depresivo, psicosis, trastorno adaptativo, alcoholismo, alcoholismo y depresión, trastorno somatomorfo y depresión, trastorno de ansiedad, toxicomanías y psicosis y sin antecedentes; intentos previos, con dos opciones de respuesta: si y no; Diagnóstico psicopatológico tras el intento con las siguientes opciones: trastorno de la alimentación, trastorno afectivo, trastorno adaptativo, trastorno de la personalidad, trastorno de ansiedad y de la alimentación, trastorno de la identidad sexual, trastorno de ansiedad, aspectos psicosociales (códigos Z de la Clasificación Internacional de Enfermedades, décima revisión CIE-10) y sin diagnóstico; conocimiento previo sobre el suicidio, con dos opciones: si y no; tipo de intento: autolítico o conducta parasuicida; topografía del intento, con tres niveles: ingesta masiva de fármacos, autolesiones y amenazas verbales; aviso de que se va a producir el intento con dos opciones: con aviso y sin aviso; motivo expresado del intento, con seis niveles de respuesta: ruptura de pareja, problemas familiares, problemas personales, relaciones interpersonales, insatisfacción personal y búsqueda de atención; existencia de pareja estable, con dos opciones: si o no; puntuación en la escala EEAG (Escala de Evaluación de la Actividad Global) del Eje V del DSM-IV-TR, es un escala con unas puntuaciones comprendidas entre el 0 y el 100, en la que las más cercanas a 100 representan una actividad satisfactoria adecuada a pautas normativas, mientras que las cercanas a 0 señalan a un sujeto con síntomas graves y con peligro de acto suicida consumado. También se recoge la respuesta de la familia ante el intento, con dos niveles: mala o buena; riesgo del intento con tres niveles: bajo, moderado y alto; tipo de tratamiento: farmacológico, psicológico, mixto y sin tratamiento; tiempo que dura el tratamiento, con cuatro niveles de respuesta: menos de seis meses, entre seis meses y un año, más de un año o 
abandona el tratamiento; día de la semana en el que se produce el intento; y mes del año en el que se produce el intento.

\section{Resultados}

A continuación se presentan los resultados descriptivos de la investigación, los estadísticos básicos y las representaciones gráficas de las variables más relevantes.

Sexo: La muestra está formada por 6 varones y 27 mujeres. En general existe una mayor frecuencia de tentativas en las mujeres, con un $82 \%$ del total de la muestra, con una proporción mayor de 4,5 a 1 .

Edad del intento: Los datos descriptivos de esta variable se resumen en la tabla 1 y quedan representados en la gráfica 1. A destacar un sujeto que presenta una tentativa suicida a los 7 años de edad, siendo el rango de mayor riesgo entre los 15 y 17 años (un $40 \%$ ), disminuyendo de forma significativa en la edad infantil, y permaneciendo estable en el tramo entre los 18 y 24 años de edad.

Nivel de estudios/ocupación: Los datos de esta variable están resumidos en la tabla 2. Destacan los porcentajes obtenidos en primaria y secundaria que representan el $66 \%$ del total de la muestra (33,3 para cada condición).

Número de hermanos: la media de hermanos que tienen los sujetos es de 2,79 y desviación típica de 1,02. El rango de hermanos en el estudio es de 1 a 5 . Las familias con mayor representación son las de 2 y 3 hermanos que representan el 75 $\%$ del total de la muestra.

Estado civil de los padres: Las parejas de padres casados representan un $81,8 \%$ (con una frecuencia de 27 ), frente a otras situaciones (viuda/o, separado/a, divoricado/a) que representan un 18,2\% (6 casos).

Antecedentes familiares psicológicos/psiquiátricos: Los datos quedan resumidos en la tabla 3. Destacan los porcentajes de sujetos con antecedentes familiares de psicopatología $(51,5 \%)$ siendo los grupos diagnósticos más representativos los trastornos afectivos y la dependencia alcohólica. Estos resultados indican que en uno de cada dos casos se valora la existencia de antecedentes psiquiátricos en los familiares

Intentos previos: Aparecen un $57,6 \%$ de casos (19 sujetos) sin antecedentes previos de intento frente a un $27,3 \%$ (9 sujetos) en los que habían realizado intentos previos. No disponiendo de información fiable en 5 sujetos que representan un 15,2\% de los casos.

Diagnóstico psicopatológico tras el intento: Los datos quedan resumidos en la tabla 4. Destaca la presencia de trastornos afectivos $(39,4 \%)$ y los sujetos sin patología $(21,2 \%)$ y trastornos adaptativos $(12,1)$.

Conocimiento previo o información sobre el suicidio: El 36,4\% de los sujetos no tiene conocimiento previo de conductas suicidas (12 casos) en familiares o entorno próximo, el $24,2 \%$ si lo tiene (8 casos) y en el 39,4\% de los casos se desconoce esta información, no está recogida en la historia clínica.

Tipo de tentativa: En un 69,7 $\%$ de las ocasiones (23 sujetos) se trata de una conducta parasuicida frente a un $30,3 \%$ (10 sujetos) que es un intento autolítico.

Topografía del intento: En un 84,8 $\%$ de los casos ( 28 sujetos) se realiza a través 
de la ingesta masiva de fármacos, en un 6,1 $\%$ ( 2 casos) por autolesiones y en un 9,1\% (3 casos) con amenazas verbales.

Aviso: En un 63,6 \% de las ocasiones (21 sujetos) se producen avisos a familiares y a allegados sobre la intencionalidad de realizar el intento, frente a un $36,4 \%$ (12 sujetos) que no avisan sobre su intención autolítica o parasuicida.

Motivo expresado: Los datos de esta variable se resumen en la tabla 5 . Destacan las rupturas de pareja $(33,3$ $\%)$ y los problemas familiares $(33,3 \%)$ como desencadenantes más frecuentes, aunque las dificultades en las relaciones interpersonales presenta también un porcentaje considerable (15,2\%).

Pareja: No tienen pareja estable el 33,3\% de los sujetos (11 casos), si la tienen el 51,5\% (17 casos) y no se dispone de datos en 5 casos (15,2\%).

Puntuación EEAG del Eje V del DSM-IV-TR: la puntuación media obtenida por los sujetos es de 80,00, con una desviación típica de 11,25 y un rango de 50 (mínimo 45 y máximo de 95).

Respuesta familiar al intento: la respuesta es buena en un $36,4 \%$ de los casos, regular en un 24,2 \% y mala en un $24,2 \%$. No disponiendo de esta información en 5 casos (15,2\%).

Valoración del riesgo: Es alto en un $12,1 \%$ (4 sujetos) de los casos, moderado en un $21,2 \%$ ( 7 sujetos) y bajo en un $66,7 \%$ (22 sujetos).

Tipo de tratamiento: Exclusivamente farmacológico en un 27,3\% de los casos (9 sujetos), solo psicológico en un 42,4\% (14 sujetos), mixto (farmacológico y psicológico) en un 24,2\% (8 sujetos), estando un $6,1 \%$ (2 sujetos) sin recibir tratamiento.
Tiempo de tratamiento: Los resultados quedan resumidos en la gráfica 2. Destacan el porcentaje de sujetos que permanecen en tratamiento menos de 6 meses $(36,4 \%)$ y el porcentaje de abandono del tratamiento $(21,2 \%)$.

Día de la semana en el que se produce el intento: Los resultados quedan resumidos en la gráfica 3. Destaca la cierta homogeneidad del intento en los diferentes días de la semana, tan solo el viernes y el sábado presentan un porcentaje significativo de no ocurrencia del intento, siendo los días más frecuentes los lunes, viernes y domingos (para cada uno de los tres un $18,2 \%$ ).

Mes del año en el que se produce el intento: Los principales resultados están resumidos en la gráfica 4. Destaca la franja de meses de verano (junio, julio, agosto y septiembre) que coincide con la temporada vacacional de los sujetos $(12,1$ $\%, 12,1 \%, 15,2 \%$ y $12,1 \%$ respectivamente). El mes en el que más intentos se producen es agosto. Señalar que no se poseen datos para un importante porcentaje de sujetos $(24,2 \%)$.

\section{Discusión}

El objetivo de este estudio es el análisis de los factores asociados a la tentativa suicida, la forma en la que se lleva a cabo y contrastar si en la población de estudio se obtienen los mismos resultados que los encontrados en la literatura científica sobre este tema. La identificación de sujetos en situación de riesgo de realizar una tentativa suicida o de repetirla, correspondería a una actividad propia de un nivel de prevención secundaria y terciaria, este tipo de prevención es fundamental para el abordaje 
de una problemática tan controvertida como es el suicidio.

Los resultados indican una mayor presencia de mujeres en las tentativas suicidas, el $82 \%$ del total de la población, dato que coincide con la mayoría de los estudios que señalan como población de mayor riesgo en la realización de tentativas suicidas a las mujeres (20). Respecto a la edad, el rango de mayor riesgo es entre los 15-17 años para nuestra muestra de estudio. En los grupos de edad inferiores nos encontramos un sujeto de 7, 11 y 12 años respectivamente. En estos casos la topografía de la tentativa consiste en amenazas verbales de hacerse daño, acto considerado como gesto suicida por los servicios de urgencias que los atendieron por lo que solicitaron consulta especializada. Este dato también coincide con la mayoría de las investigaciones donde el riesgo se incrementa con la edad, siendo extremadamente raros los casos de suicidio consumado en menores de 12 años, resultando más frecuente para este tramo de edad las conductas parasuicidas (14). Sin embargo, en esta investigación encontramos un sujeto con tan solo 7 años que presenta un gesto suicida. No sabemos lo excepcional del caso o si quizás el descenso en la edad de los intentos es una tendencia actual ante la que debemos estar alerta y estudiar con mayor detenimiento.

En cuanto al nivel de estudios, en la mayoría de los casos encontramos un bajo rendimiento académico, siendo lo más frecuente los estudios primarios y secundarios, que no correspondería con la distribución por edad de la muestra, lo que indica que han repetido curso y/o han abandonado los estudios en un alto porcentaje. En relación a esta cuestión Mardomingo (29), señala que en un $25 \%$ de los casos estudiados no terminan los estudios primarios, alcanzando un nivel educativo inferior comparado con las pautas normativas de su población de referencia.

Los resultados encontrados en relación a la situación familiar denotan que en la mayoría de los casos los padres de los menores están casados, quizás la valoración de la calidad de la relación parental aportaría datos para un mejor análisis pero la información recogida en las historias clínicas no recoge datos exhaustivos de este tipo de relaciones.

Un dato significativo es que en más de la mitad de los casos hay presencia de antecedentes psiquiátricos en los padres, resultado que es inespecífico para el suicidio ya que se presenta también en otros problemas sociales o patologías psicológicas, pero que en este caso está muy representado, siendo un dato que se repite en los estudios sobre el tema (32).

Los trastornos psiquiátricos más frecuentes dentro de los antecedentes familiares son los problemas afectivos y las toxicomanías, estos resultados coinciden con los señalados por otros autores (32) que indican la proliferación de diversas psicopatologías en el núcleo familiar al que pertenece la persona que realiza el intento.

Respecto a la valoración de presencia de psicopatología tras el intento, encontramos que los trastornos afectivos y los trastornos adaptativos con sintomatología ansioso-depresiva, son los diagnósticos más frecuentes en la muestra que participa en este estudio. En esta línea, numerosos investigadores consideran 
que los trastornos afectivos también son los diagnósticos psiquiátricos más frecuentes, Marttunen y colaboradores (33) encontraron una prevalencia del $51 \%$ y Brent y colaboradores (34) informaron que el diagnóstico de trastorno afectivo se producía en el $49 \%$ de los adolescentes suicidas. Estos resultados se repetían también en las tentativas aunque hay mayor confusión entre síntomas y síndromes para este grupo (4). Otro resultado relevante es el alto porcentaje que se considera sin patología representando un $22 \%$ de la muestra de este estudio, este dato contrasta con los obtenido en investigaciones sobre suicidio consumado que señalan que solo un bajo porcentaje estaría libre de un diagnostico psiquiátrico y que en las tentativas suicidas se encontrarían un menor porcentaje de trastornos psiquiátricos, sobre el $80 \%$ (33), resultado que coinciden con los obtenidos en el presente estudio. En esta línea, los datos de una menor tasa de psicopatologia en las tentativas estarían asociados más a un intento suicida de tipo impulsivo, con determinadas características de personalidad como reacciones emocionales intensas, escasa tolerancia a la frustración, sentimiento de cólera, dificultad para demorar la gratificación, estilo cognitivo rígido, impulsividad, escasa flexibilidad ante las demandas ambientales y deficiente imagen personal (35).

Un dato a destacar es la presencia de un $30 \%$ de intentos previos, porcentaje muy alto para una población tan joven, hecho que indica el riesgo de cometer una tentativa suicida por este grupo de población, siendo a mayor número de intentos de autolisis previos mayor el riesgo de suicidio. En esta línea, según De
Wilde y colaboradores (1), afirman que haber realizado una conducta del espectro suicida está considerado como un factor de riesgo para repetir la tentativa suicida o para que esta pueda consumarse, este hecho toma mayor relevancia en la infancia y adolescencia donde se ha observado un incremento en las últimas décadas de las tentativas suicidas, identificando la adolescencia como una edad de máximo riesgo (1).

El tipo de acto suicida más frecuente es la conducta parasuicida, la valoración del riesgo por el clínico que atiende la urgencia es baja, respecto a su letalidad, en la mayoría de los sujetos, siendo la valoración del riesgo alta en menos de un tercio de los casos. Estos datos están íntimamente relacionados con la creencia de que las llamadas de atención y la obtención de beneficios secundarios están detrás de un gran número de comportamientos parasuicidas llevados a cabo por menores.

En relación a esta cuestión, respecto a la topografía de la tentativa, en la mayoría de las ocasiones, ha sido la ingesta medicamentosa acompañada en numerosas ocasiones de alguna conducta de aviso por parte del sujeto la que con más frecuencia se presenta. Un método de baja letalidad y con posibilidades de rescate no indica en sí mismo ausencia de gravedad, además debemos tener en cuenta que un elevado número de sujetos no conocen los efectos derivados de estas conductas. Un análisis del acto como una forma de llamada de atención, una "rabieta" o un modo de manipulación, es un abordaje simplista de la situación y resulta perjudicial para el abordaje terapéutico ya que toda tentativa suicida 
indicaría una situación de desajuste personal y una falta de recursos personales de afrontamiento. La realización de un análisis detallado y de la valoración de la psicopatología asociada prevendría el riesgo de repetir la tentativa.

Respecto a la atención que se presta en nuestra unidad (sin definir el tipo de abordaje que daría para otra investigación) para las tentativas suicidas en un $45 \%$ de los casos se realiza una intervención psicológica, en un $25 \%$ de los casos una intervención mixta, en un $25 \%$ exclusivamente psicofarmacológica y en un 5\% (tan solo dos casos) no se valoró necesario la realización de tratamiento continuado.

En esta línea, la estancia media en el dispositivo de atención se distribuye entre un $35 \%$ que está menos de 6 meses, un $35 \%$ en torno al año y en un $20 \%$ los que abandonan sin el visto bueno del facultativo, porcentaje significativamente superior a la media de nuestra unidad que se sitúa alrededor del $12 \%$ para el total de las consultas. Estos datos alertan sobre un número importante de abandonos que en posteriores estudios longitudinales sería interesante contrastar si vuelven a presentar intento autolítico o conducta parasuicida.

El motivo desencadenante expresado por los sujetos, (que en si mismo no es suficiente para provocar la conducta suicida, pero que en combinación con otros factores puede precipitarla) indican que el fracaso en las relaciones interpersonales (ruptura de pareja), los problemas familiares y la separación del grupo de amigos son los principales desencadenantes que explicarían aproximadamente el 80 por ciento de los casos producidos. No encontramos en nuestra muestra el fracaso académico como desencadenante, hecho que si aparece en otros estudios $(32,9)$. Los jóvenes le dan un mayor peso a las relaciones sociales y familiares, hecho que en la adolescencia toma un valor primordial, siendo el miedo a la soledad uno de los temores principales de los jóvenes.

Respecto a las variables que recogen el mes y día de la semana en el que ocurre el intento, estas señalan que la mayoría de los casos se producen en los meses de verano, distribuyéndose de forma homogénea a lo largo de los diferentes días de la semana a excepción del viernes y el sábado en los que se produce una frecuencia bastante inferior. En comparación con el resto de los días. Estos datos también se repiten en las estadísticas sobre suicidio $(26,18)$, mostrando picos en los meses de primavera y verano. Respecto al día de la semana, también existe coincidencia en señalar los días intermedios como los más frecuentes en la producción de intentos siendo los fines de semana los días de menor ocurrencia. Sin embargo, en nuestros resultados, si bien ningún sujeto realiza el intento en viernes y tan solo uno en sábado, el domingo si es un día de los que presenta mayor frecuencia de intentos.

En conclusión, el estudio de los resultados de las principales variables analizadas en la literatura especializada sobre el tema, coinciden con los obtenidos en este estudio en líneas generales. No obstante algunos resultados que destacan en este trabajo son el decremento en la edad del intento, con un caso que incluso realiza la tentativa a una edad de 7 años, que nos hace plantearnos la posibilidad de 
estar produciéndose un descenso progresivo en la edad de los sujetos. La ausencia de psicopatología en un elevado número de casos. Y la proliferación de intentos en domingo, son los resultados más destacados si los comparamos con los obtenidos en la literatura especializada sobre el tema.

Finalmente, según los resultados obtenidos podríamos indicar los principales rasgos que conforman un perfil tipo de las tentativas suicidas de nuestra población de estudio, este sería el de una mujer adolescente, con familiares con antecedentes psiquiátricos, con intentos autolíticos previos y en el medio cercano, con bajo rendimiento académico, presencia de psicopatología afectiva, con un conflicto relacional como desencadenante, realizando una tentativa suicida mediante sobreingesta de fármacos con conducta de aviso.

Tabla 1. Estadísticos básicos de la variable "edad del intento"

\begin{tabular}{ll}
\hline Media & 17,1 \\
Mediana & 17,0 \\
Moda & 16 \\
Desviación Típica & 3,7 \\
Varianza & 13,8 \\
Rango & 17 \\
Mínimo & 7 \\
Máximo & 24 \\
\hline
\end{tabular}

Gráfica 1. Distribución de frecuencias de la edad en la que se produce el intento

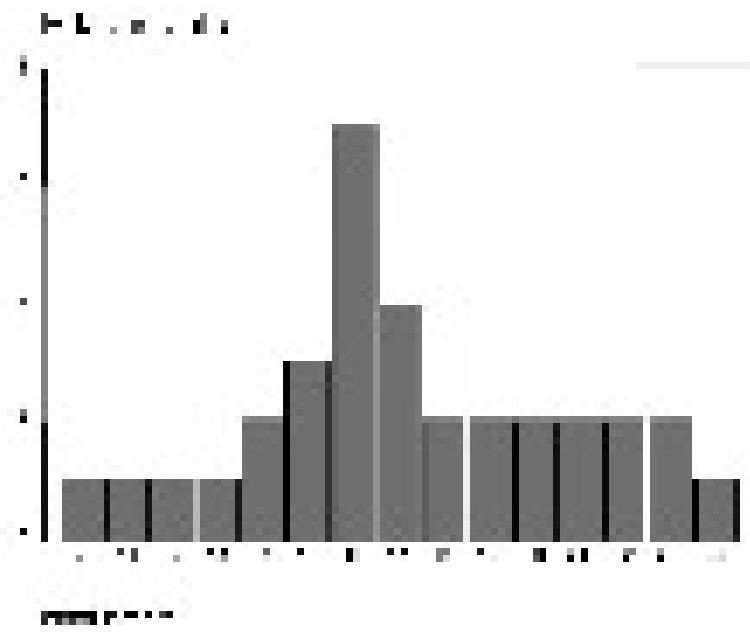


Tabla 2. Frecuencias y porcentajes de los sujetos según nivel de estudios/ocupación

\begin{tabular}{lcc}
\hline & Frecuencia & Porcentaje \\
\hline Primaria & 11 & 33,3 \\
Secundaria & 11 & 33,3 \\
Bachiller & 4 & 12,1 \\
FP & 2 & 6,1 \\
Universidad & 4 & 12,1 \\
Trabajo & 1 & 3,0 \\
\hline Total & 33 & 100,0 \\
\hline
\end{tabular}

Tabla 3. Frecuencias y porcentajes de sujetos según antecedentes familiares psicológicos/ psiquiátricos

\begin{tabular}{lcc}
\hline & Frecuencia & Porcentaje \\
\hline Trastorno Depresivo & 6 & 18,2 \\
Alcoholismo & 1 & 3,0 \\
Psicosis & 3 & 9,1 \\
Trastorno Adaptativo & 1 & 3,0 \\
Alcoholismo y Depresión & 3 & 9,1 \\
T. Somatomorfo y Depres. & 1 & 3,0 \\
Toxicomanías y Psicosis & 1 & 3,0 \\
Trastorno de Ansiedad & 1 & 3,0 \\
Sin Antecedentes & 16 & 48,5 \\
\hline Total & 33 & 100,0 \\
\hline
\end{tabular}

Tabla 4. Frecuencias y porcentajes de sujetos según la variable diagnóstico psicopatológico tras el intento

\begin{tabular}{lcc}
\hline & Frecuencia & Porcentaje \\
\hline Trastornos Afectivos & 13 & 39,4 \\
Trastornos Alimentación & 2 & 6,1 \\
Trastorno Adaptativo & 4 & 12,1 \\
Trastorno Personalidad & 2 & 6,1 \\
Trastorno Id.. Sexual & 1 & 3,0 \\
Trastorno Ansiedad & 1 & 3,0 \\
T. Ansiedad y Alimentación & 1 & 3,0 \\
Aspectos psicosociales & 2 & 6,1 \\
Sin Patología & 7 & 21,2 \\
\hline Total & 33 & 100,0 \\
\hline
\end{tabular}


ORIGINALES Y REVISIONES

Tabla 5. Frecuencias y porcentajes de sujetos según la variable motivo expresado

\begin{tabular}{lcc}
\hline & Frecuencia & Porcentaje \\
\hline Ruptura Pareja & 11 & 33,3 \\
Problemas Familiares & 11 & 33,3 \\
Problemas Personales & 3 & 9,1 \\
Insatisfacción Personal & 2 & 6,1 \\
Búsqueda de Atención & 1 & 3,0 \\
Relaciones interpersonales & 5 & 15,2 \\
\hline Total & 33 & 100,0 \\
\hline
\end{tabular}

Gráfica 2. Frecuencia de sujetos según tiempo que permanecen bajo tratamiento

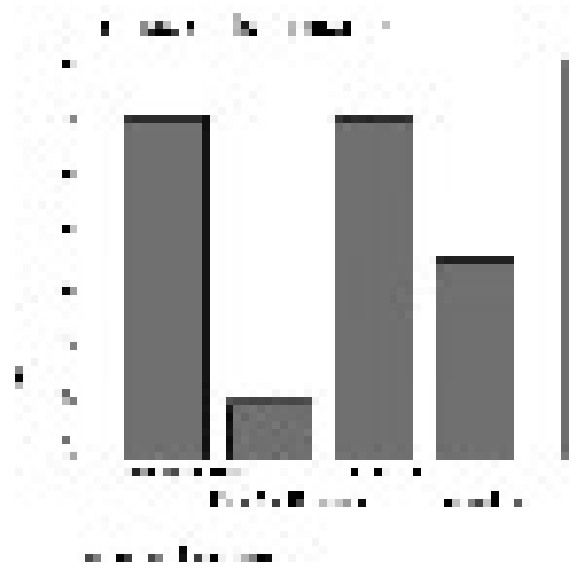

Gráfica 3. Frecuencia de sujetos según el día de la semana en el que se produce el intento

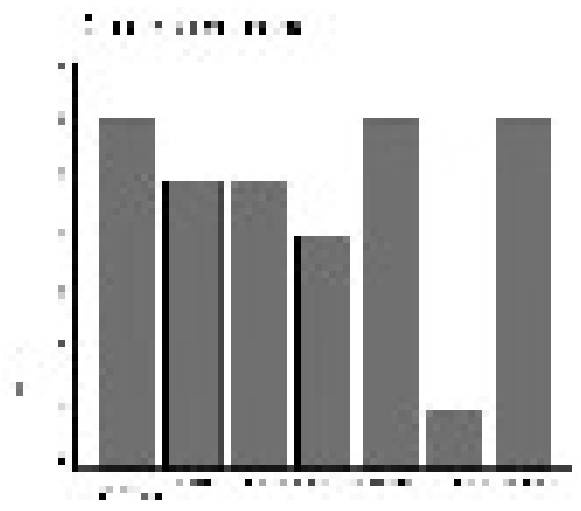


Gráfica 4. Número de sujetos según el mes en el que se produce el intento

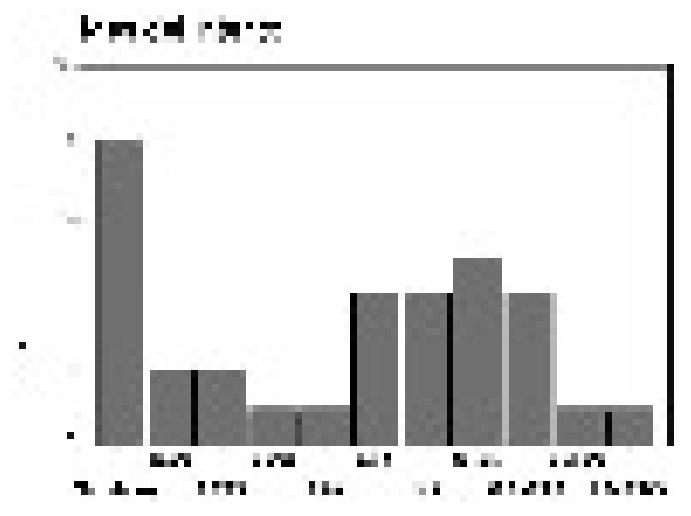

\section{BIBLIOGRAFIA}

1. De Wilde; E.J., Kienhorst, I.C.; Diekstra, R.F.W. "El suicidio en la adolescencia”. En J. Buendía (Ed.) Psicopatología en niños y adolescentes. Madrid, Pirámide, 1996.

2. González, C.; Berenzon, S.; Jiménez, A. "Al borde de la muerte: problemática suicida en adolescentes”. Salud Mental, 1999, Número Especial.

3. Mardomingo M..J.; Catalina, M.L. "Suicidio e intentos de suicidio en la infancia y en la adolescencia: ¿un problema en aumento?”. Pediatrika, 1992, 12, 118-121

4. González J.C. y otros. "Factores psicológicos y psicopatológicos". En J. Bobes, Gozalez J.C.; Sáiz P.A. (Eds), Prevención de las conductas suicidas y parasuicidas (pp. 59-68). Barcelona, Masson, 1997.

5. Ruiz, J.A.; Riquelme, A.; Buendia, J. "Personalidad y comportamiento suicida en adolescentes: El papel de la extraversion en la tentativa de suicidio". Clínica y Salud, 2000 11, 155-169.

6. Viñas, F.; Jane, M.C.; Domenech, E. "Evaluación de la severidad de la ideación suicida autoinformada en escolares de 8 a 12 años". Psicothema, 2000, 12, 594-598.

7. Leenaars, A. y otros. "Suicide notes of adolescentes: A life-span comparision". Canadian Journal of Behavioural Science, 2001 33, 47-57.

8. Pfeffer C.R. The suicidal child. New York, Guilford Press, 1986.

9. Pfeffer C.R. "Suicidal Behavior in children and adolescents: causes and management". En: Lewis M. (Ed). Child and Adolescent psychiatry. A comprensive testbook. 2 end. ed. Baltimore, Williams and Wilkins, 1996.

10. Carlson, G.A.; Cantwell, D.P., "Suicidal Behaviour and depression in children and adolescents". Journal American Academy of Child Psychiatry, 1982, 21, 361-368. 
11. Mardomingo M. J. Psiquiatría del niño y del adolescente. Madrid, Díaz de Santos, 1994.

12. Goldney, R.D. y otros. "Suicidal ideation in a young adult population". Acta Psychiatrica Scandinavica, 1989, 79, 481-489.

13. Choquet M.; Facy F.; Davidson, F. Suicide and attempted suicide amoog adolescents in France. The suicide syndrome. London, Croom Helm Ltd, 1980.

14. Gastaminza, X.; Venteo, P.; Tomas, J. "Las tentativas de suicidio en la infancia y en la adolescencia: aproximación paramétrico epidemiológica". Revista de Neuropsiquiatria Infantil, 1983, 2, 123-155.

15. INE. Estadística del suicidio para el quinqenio1996- 2001. Madrid, INE, 2002.

16. Arranz, F.J. "Factores psicologicos-conductuales y psicosociales de riesgo suicida. En Ros Montalban", S. (Ed.) La Conducta Suicida (pp. 81-96) Madrid, Editorial Libro del Año, 1997.

17. Blumenthal, S.J.; Kupfer D.J., (1986) "Generalizable treatment strategies for suicidal behavior". Annals of the New York Academy of Sciences, 1986, 487, 327-340.

18. González M.P. y otros. "Factores sociodemográficos". En J. Bobes; González J.C.; Sáiz P.A. (Eds), Prevención de las conductas suicidas y parasuicidas (pp. 46-54). Barcelona, Masson, 1997.

19. Balanza, J.; Chamorro, L. "Analisis del suicidio consumado en Guadalajara. Aspectos epidemiologicos y perfiles suicidas. Archivos de neurobiologia, 1999, 62, 163-176.

20. Canetto S.S. "Gender and suicidal behavior. Theories and evidence". En: Maris R.W.; Silverman M.M; Canetto S.S., eds., Review of Suicidology. New York, The Guilford Press, 1997.

21. Irigoyen, I. y otros. "Características y prevalencia del suicidio consumado en población rural". Psiquiatria pública, 1997, 9, 105-116.

22. Martí, G.; Corbella, J. "Evolución del suicidio consumado en Barcelona”. Jano, 1988, 2, 27-31.

23. Adam, K.S. "Enviromental, psychosocial, and psychoanalitic aspects of suicdal behavio"r. En: Blumenthal S.J.; Kupfer, D.J.. (Eds). Suicide over the life cycle. Washington, American Psychiatric Press, 1990.

24. Fernández, A.; González, M.A.; Lasa, A. "Tentativas de suicidio en jovenes adultos: estudio de los acontecimientos vitales recientes y eventos precipitantes”. Psiquis, 1996, 17, 51-58.

25. Moscicki, E.K. "Epidemiology of suicidal behaviour". Suicide and life threattening behavior, $1995,25,22-35$

26. Sarró, B.; Surribas C.; De la Cruz C. "Suicidio, imitación e información”. Jano, 1988, 2, 61-64.

27. Mardomingo M.J.; Catalina M.L.; Hernández P. Curso Clínico de los intentos de suicidio: Seguimiento longitudinal a lo largo de ocho años. Libro de ponencia VII Congreso de psiquiatría infanto-juvenil, 71-78, 1994.

28. Wichstrom, L. "Predictors of adolescente suicide attempts: A nationally representative longitudinal study of Norwegian adolescents". Journal of the American Academy of child and adolescent Psychiatry. 2000, 39 , 603-610. 
29. Mardomingo M.J. "Suicidio y tentativas autoliticas". En J.R. Gutiérrez Casares; F. Rey Sánchez (Coordinadores), Planificación terapéutica de los trastornos psiquiátricos del niño y del adolescente (pp.1195-1232). Madrid, Smithkline Beecham, 2000.

30. Leisering, D.K. "Rural youth suicide prevention: An evaluation of a community based intervetion project". Dissertation Abstracts International Section-B. 2000, 60 (7-B), 3570.

31. Saiz, P.A.; Cocaña I.; González M.P. Psychological evaluation in parasuicide behaviour. X World Congress of Psychiatry. Madrid, 1996.

32. González-Seijo J.C. y otros. "Factores familiares en las tentativas de suicidio de adolescentes". Actas luso-españolas neurología, psiquiatría y ciencias afines, 1996, 24, 12-18.

33. Marttunen M.J. y otros. "Mental disorders in adolescent suicide. DSM-III-R Axes I and II Diagnoses in suicides among 13 to 19 years olds in Finlad". Archives General Psychiatry, 1991, 48, 834-839

34. Brent D.A. y otros. "Psychiatric risk factors for adolescents suicide: a case control study". Journal American Academy Child Adolescent Psychiatry, 1993, 32, 521-529.

35. Borst S.R.; Noam G.G. "Suicidality and psychopathology in hospitalized children and adolescents". Acta paedopsychiatry, 1989, 52, 165-175.

*Psicólogo Clínico. Equipo de Salud Mental Distrito Montilla. (Hospital Universitario Reina Sofía, Córdoba)

**Profesor Universidad de Córdoba. Área de Personalidad, Evaluación y Tratamiento Psicológicos, Facultad de Ciencias de la Educación.

Correspondencia: José Fernando Venceslá Martínez. C/ Daza de Valdés nº $61^{\circ} 14006$ Córdoba. Telf. 957-405638. E-mail VENCESLA@ grupobbva.net

Recibido: 08-07-02 